\title{
Neuromodulation by non-invasive brain stimulation (NIBS): a step back to move forward
}

\author{
MIGUEL FERNANDEZ-DEL-OLMO1 | MARTA SEVILLA-SANCHEZ² | GONZALO MARQUEZ-SANCHEZ² | DAWSON \\ KIDGELL'3 | MARIE-HELENE MILOT ${ }^{4}$ | RUUD W. SELLES ${ }^{5,6}$ | JOHN ROTHWELL ${ }^{7}$ TIBOR HORTOBÁGY ${ }^{8}$ \\ ${ }^{1}$ Area of Sport Sciences, Faculty of Sports Sciences and Physical Education, Center for Sport Studies, King Juan Carlos University, Madrid, Spain. \\ 2Department of Physical Education and Sport, Faculty of Sports Sciences and Physical Education, University of A Coruna, A Coruna, Spain. \\ 3Department of Physiotherapy, School of Primary and Allied Health Care, Faculty of Medicine, Nursing and Health Science, Monash University, Melbourne, Australia. \\ ${ }^{4}$ Centre de recherche sur le vieillissement, École de réadaptation, Faculté de médecine et des sciences de la santé, Université de Sherbrooke, Sherbrooke, QC, Canada. \\ 5Department of Rehabilitation Medicine, Erasmus MC, University Medical Centre Rotterdam, Rotterdam, The Netherlands. \\ ${ }^{6}$ Department of Plastic and Reconstructive Surgery, Erasmus MC, University Medical Centre Rotterdam, Rotterdam, The Netherlands. \\ ${ }^{7}$ Sobell Department of Motor Neuroscience and Movement Disorders, University College London, Institute of Neurology, London, United Kingdom. \\ ${ }^{8}$ Center for Human Movement Sciences, University Medical Center Groningen, The University of Groningen, Groningen, The Netherlands.
}

Correspondence to: Miguel Fernandez del Olmo - Area of Sport Sciences, Faculty of Sports Sciences and Physical Education - Center for Sport Studies - King Juan

Carlos University - Camino del Molino, s/n, 28943 Fuenlabrada, Madrid, Spain.

email: miguel.delolmo@urjc.es

https://doi.org/10.20338/bjmb.v15i2.213

\section{ABBREVIATIONS \\ iTBS Intermittent theta burst (magnetic) stimulation \\ M1 primary motor cortex \\ NIBS non-invasive brain stimulation \\ rTMS repetitive transcranial magnetic stimulation \\ tDCS transcranial direct current stimulation}

The first studies on non-invasive brain stimulation (NIBS) appeared barely 20 years ago showing lasting changes in cortical excitability of motor areas following repetitive transcranial magnetic stimulation (rTMS) $)^{1}$ and transcranial direct current stimulation (tDCS). ${ }^{2}$ These pioneering studies opened up new vistas for the exploration of treatment alternatives for diverse pathologies (from depression to joint instability) and for the enhancement of cognitive and motor function. Indeed, the $\sim 2,000$ papers published on NIBS during the past two years is a resolute expression of the effort to fully develop these techniques of cortical stimulation for quick, customized, reliable, lasting, and most importantly, corrective modulation of putative brain circuits underlying neurological, orthopedic, behavioral, and cognitive impairments.

As a result of this immense research effort, NIBS-induced neuromodulatory treatments are now considered 'evidence-based' and have become readily available at clinics and rehabilitation centers. With exception of rTMS for treating depressive symptoms, recommendation for therapeutic use of NIBS is based mainly on meta-analytical estimates of NIBS effects observed in studies using very small and heterogenous samples, and without randomization, blinding to treatment, or placebo stimulation. The aim of the current opinion is not to cast a critical light on the NIBS literature. Rather, we wish to reflect on key features of the purported neuromodulatory effects of NIBS, i.e., the inconsistency in inducing changes in brain excitability and replicating such changes induced by NIBS.

Depending on the nature (magnetic, electrical) and the wave properties of the stimulus, NIBS can increase or decrease the excitability of circuits targeted. Intermittent 
theta burst (magnetic) stimulation (iTBS) has excitatory after-effects on the primary motor cortex (M1). However, several studies, using various rTMS protocols, failed to observe the expected rise in excitability, while others showing increased excitability revealed that the effects were unreliable.,4 The interneuron networks targeted, shape of the stimulus waveform, time of day, attention, and genetics are just some of the factors that could contribute to the inconsistencies between studies. A further complicating factor is that NIBS can have lasting effects, as was the case after rTMS at $1 \mathrm{~Hz}$, which tends to decrease M1 excitability. When the first rTMS session was repeated after 5 days, there was a cumulative effect of a deepened M1 inhibition, making session-to-session reliability assessments questionable. ${ }^{5}$

Inter-individual differences in the required current intensity, direction, and the size of the electric field of NIBS could underlie responsiveness differences across individuals, potentially contributing to its unreliability. The large inter-individual variation and poor responsiveness to NIBS was evident after 56 healthy adults received paired associative stimulation, anodal tDCS, and iTBS, but only $\sim 45 \%$ of participants exhibited changes in M1 excitability after each protocol and only $12 \%$ responded to all three. ${ }^{6}$ Even these response rates are questionable because the NIBS effects were not compared with sham controls, leaving the 'true' effects undetermined. Therapeutic NIBS effects are believed to rise with increasing number of sessions, although evidence is inconclusive for such cumulative effect following repeated NIBS sessions, ${ }^{7}$ even if the trial comprises a large sample, randomization, placebo-control, and double blinding. ${ }^{8}$ Brain health state seems to further increase inter-individual variability in responsiveness to NIBS. For instance, it augmented the effects of motor training on healthy older adults' functional outcomes, but not in individuals at the chronic phase of a stroke following paretic arm strength training. 9,10

The emerging perspective is that NIBS holds promise to become an independent treatment but, perhaps more likely, as an adjuvant to standard rehabilitation. There is a need to perform randomized, double-blind, and sham-controlled trials in large sample sizes with pre-registration of the protocol. Future studies may need to better control the NIBS application, as for example by including EEG-controlled stimulation and an accurate modelling of the current flow in the brain. In addition, in many disease applications, we need a better understanding of the underlying mechanisms of the brain networks that NIBS aims to modulate and take this into account when designing a personalized NIBS application. Such approaches could account for variations in brain anatomy and increase targeting accuracy of the magnetic or electric field, which could then increase the number of responders, reduce the variation between responders, and produce acceptable reliability within and between sessions.

Only by taking a step back can we ensure that these promising interventions improve neurological and cognitive impairments through a 'real' neuromodulatory effect.

\section{REFERENCES}

1. Chen R, Classen J, Gerloff C, Celnik P, Wassermann EM, Hallett M et al. Depression of motor cortex excitability by low-frequency transcranial magnetic stimulation. Neurology. 1997;48(5):1398-403. doi: 10.1212/WNL.48.5.1398

2. Nitsche MA, Paulus W. Sustained excitability elevations induced by transcranial DC motor cortex stimulation in humans. Neurology. 2001;57(10):1899-901. 
doi: 10.1212/wnl.57.10.1899

3. Ji GJ, Wei JJ, Liu T, Li D, Zhu C, Yu F et al. Aftereffect and Reproducibility of Three Excitatory Repetitive TMS Protocols for a Response Inhibition Task. Front Neurosci. 2019;13:1155. doi: 10.3389/fnins.2019.01155

4. Hamada M, Murase N, Hasan A, Balaratnam M, Rothwell JC. The role of interneuron networks in driving human motor cortical plasticity. Cereb Cortex. 2013;23(7):1593-605. doi: $10.1093 /$ cercor/bhs147

5. Hortobágyi T, Richardson SP, Lomarev M, Shamim E, Meunier S, Russman H et al. Chronic low-frequency rTMS of primary motor cortex diminishes exercise training-induced gains in maximal voluntary force in humans. J Appl Physiol (1985). 2009;106(2):403-11. doi: 10.1152/japplphysiol.90701.2008

6. Lopez-Alonso V, Cheeran B, Rio-Rodriguez D, Fernandez-Del-Olmo M. Inter-individual variability in response to non-invasive brain stimulation paradigms. Brain Stimul. 2014;7(3):372-80. doi: 10.1016/j.brs.2014.02.004

7. Perellon-Alfonso R, Kralik M, Pileckyte I, Princic M, Bon J, Matzhold C et al. Similar effect of intermittent theta burst and sham stimulation on corticospinal excitability: A 5-day repeated sessions study. Eur J Neurosci. 2018;48(4):1990-2000. doi: 10.1111/ejn.14077

8. Jonker ZD, Gaiser C, Tulen JHM, Ribbers GM, Frens MA, Selles RW. No effect of anodal tDCS on motor cortical excitability and no evidence for responders in a large double-blind placebo-controlled trial. Brain Stimul. 2020; 4(1):100-109. doi: 10.1016/j.brs.2020.11.005

9. Beaulieu LD, Blanchette AK, Mercier C, Bernard-Larocque V, Milot MH. Efficacy, safety, and tolerability of bilateral transcranial direct current stimulation combined to a resistance training program in chronic stroke survivors: A double-blind, randomized, placebocontrolled pilot study. Restor Neurol Neurosci. 2019;37(4):333-46. doi: 10.3233/RNN190908

10. Rostami M, Mosallanezhad Z, Ansari S, Kidgell D, Rezaeian T, Bakhshi E et al. The effects of consecutive sessions of anodal transcranial direct current stimulation over the primary motor cortex on hand function in healthy older adults. Arch Gerontol Geriatr. 2020;89:104063 
Citation: Fernandez-del-Olmo M, Sevilla-Sanchez M, Marquez-Sanchez G, Kidgell D, Milot MH, Selles RW, Rothwell J, Hortobágyi T. Neuromodulation by non-invasive brain stimulation (NIBS): a step back to move forward. BJMB. 2021. 15(2): 61-64.

Editors: Dr Fabio Augusto Barbieri - São Paulo State University (UNESP), Bauru, SP, Brazil; Dr José Angelo Barela São Paulo State University (UNESP), Rio Claro, SP, Brazil; Dr Natalia Madalena Rinaldi - Federal University of Espírito Santo (UFES), Vitória, ES, Brazil.

Section Editors (Current Opinion): Dr Luis Augusto Teixeira - University of São Paulo (USP), São Paulo, SP, Brazil; Dr Tibor Hortobágyi - University of Groningen, The Netherlands; Dr Renato de Moraes - University of São Paulo (USP), Ribeirão Preto, SP, Brazil.

Copyright:@ 2021 Fernandez-del-Olmo, Sevilla-Sanchez, Marquez-Sanchez, Kidgell, Milot, Selles, Rothwell and Hortobágyi and BJMB. This is an open-access article distributed under the terms of the Creative Commons AttributionNon Commercial-No Derivatives 4.0 International License which permits unrestricted use, distribution, and reproduction in any medium, provided the original author and source are credited.

Funding: There was no funding for this study.

Competing interests: The authors have declared that no competing interests exist. DOl: https://doi.org/10.20338/bjmb.v15i2.213 